\title{
A kind of effective data aggregating method based on compressive sensing for wireless sensor network
}

\author{
De-gan Zhang ${ }^{1,2}$, Ting Zhang ${ }^{1,2}$, Jie Zhang ${ }^{3^{*}}$, Yue Dong ${ }^{1,2}$ and Xiao-dan Zhang ${ }^{4}$
}

\begin{abstract}
Wireless sensor network (WSN) in the Internet of Things consists of a large number of nodes. The proposal of compressive sensing technology provides a novel way for data aggregation in WSN. Based on the clustering structure of WSN, a kind of effective data aggregating method based on compressive sensing is proposed in this paper. The aggregating process is divided into two parts: in the cluster, the sink node sets the corresponding seed vector based on the distribution of network and then sends it to each cluster head. Cluster head can generate corresponding own random spacing sparse matrix based on its received seed vector and collect data through compressive sensing technology. Among clusters, clusters forward measurement values to the sink node along multi-hop routing tree. Performance analysis and comparison with the relative methods show that our method is effective and superior to other methods regardless of intra-cluster or inter-cluster on the total energy consumption of network.
\end{abstract}

Keywords: WSN, Internet of things, Compressive sensing, Data aggregating, Effective

\section{Introduction}

Data aggregating is an effective strategy to control energy consumption because the number of transmissions can be reduced after aggregation. Reference [1-4] strives for energy balancing to make the network lifetime maximum. The unbalanced consumption of energy is harmful to network safety and health [5-9]. If the sensor nodes of wireless sensor networks (WSNs) spend their energy in a relatively balanced way, the connectivity among sensor nodes and the sink nodes can be kept for a longer time, making the network segmentation to be postponed. Avalanched quantities of tiny sensor nodes establish WSNs in the Internet of Things. These nodes can monitor all kinds of object information around them in real-time. Since the energy of these sensor nodes is usually very limited, how to ensure complete data aggregating with the minimum energy consumption of nodes has been a very critical issue in WSNs [10-12].

In order to remove redundant portions of the collected data, and control the number of data nodes in WSNs,

\footnotetext{
*Correspondence: 1185638995@qq.com

${ }^{3}$ Beijing No. 20 High School, Xiaoyingxilu, Haidian District, Beijing 100085, China

Full list of author information is available at the end of the article
}

which can save the energy consumption of nodes, recently, many scholars proposed a compressive sensing (CS) technology, which can collect and reconstruct signal with high probability through sampling points less than the Nyquist sampling theorem [13-18]. According to the sparsity of the signal, compressive sensing technology can decrease the original signal from high dimensional to low dimensional on the nodes. It needn't aggregate the signal and recover it with high probability on the sink node. The proposal of compressive sensing has good performance on image processing and other applications [19-27].

Without using compressive sensing in data aggregation, nodes near the leaves forward a small amount of packets, but those which are close to the sink node need to forward a large number of packets [28-30]. With using compressive sensing in data aggregating, each node simply forwards $M$ packets, so the total transmission number of the network with $N$ nodes is $M N$. However, transmission quantity is still large. References [6-10] proposed a hybrid protocol. In this protocol, nodes near the leaves forward original data without using compressive sensing, and those which are close to the sink node use compressive sensing technology to transmit data. References 
[31-35] applied hybrid compressive sensing to the data aggregating and proposed a minimum energy aggregation tree. The previous work directly applies compressive sensing method to the route tree. Since clustering method has many advantages over the routing tree [36-40], compressive sensing method on clustering network is applied. Compared with routing tree data aggregating methods, clustering algorithm generally has a better communication load balance $[41,42]$. In addition, previous works ignore the distribution of location information and node distribution, which can contribute that data aggregating consumes less energy in WSNs of the Internet of Things [43-47].

References [13, 14] proposed Toeplitz matrix and proved that it meets the restricted isometry property (RIP). Since the correlation of data collected in a single cluster is relatively large, the sparse matrix to the process of compressive sensing can be used. It can minimize the number of independent random variables, which can reduce the complexity of compressive sensing process, and improve the calculation speed in the meantime.

The literature [14-18] proposed Toeplitz random measurement matrix and proved it. The literature [17-26] proposed quasi-Toeplitz matrix, semi-Hadamard matrix, and chaos-Toeplitz matrix and proved that they met the condition of the RIP. Based on the former researches, some scholars [27-35] proposed random spacing sparse Toeplitz matrix optimized by singular value decomposition (SVD) and apply it in wireless sensor networks.

According to random space sparse matrix based on the Toeplitz matrix, the vector $T_{1}=\left[\phi_{1}, \phi_{2}, \ldots, \phi_{N}, \phi_{N+1}, \ldots\right.$, $\left.\phi_{N+M-1}\right]$ contains all the elements of a Toeplitz matrix. They sparse $T_{1}$ with space $\Delta=2$, the value of its element $\phi_{i}(i \in \Lambda, \Lambda$ is $\Gamma(N+M-1) / \Delta\rceil$ indexes randomly selected from $1 \sim N+M-1)$ is subject to independent and identically distributed, and the other elements are set to 0 . Finally, the sparse vector is used to construct random spacing sparse Toeplitz matrix:

$$
\phi_{i+1, j+1}=\phi_{i, j}
$$

The Gaussian random matrix requires $M N$-independent random elements, the general Toeplitz matrix only needs $M+N-1$, and the random space sparse Toeplitz matrix needs only $\left.\lceil(N+M-1) / \Delta\urcorner\right|_{\Delta=2, \ldots, 16}$ independent random elements, so it is possible to further reduce complexity.

The innovation or contribution of this paper is as follows: based on the clustering structure of WSNs, a new data aggregating method based on sparse hybrid compressive sensing is proposed The aggregating process is divided into two parts: in the cluster, the sink node sets the corresponding seed vector based on the distribution of network and then sends it to each cluster head. Cluster head can generate corresponding own random spacing sparse matrix based on its received seed vector and collect data through compressive sensing technology. Among clusters, clusters forward measurement values to the sink node along the multi-hop routing tree which we built before. Performance analysis and comparison of the experimental results with the relative methods show that our method is effective and superior to other methods regardless of intra-cluster or inter-cluster on the total energy consumption of network and the lifetime of network.

\section{Modeling based on hybrid compressive sensing for WSN}

In the data aggregating process, first of all, the network is clustered. Each cluster has its cluster head, one sample is shown in Fig. 1. The measurement matrix of the entire network is generated by sink nodes according to the sparse seed vector and sends the sparse seed vector to each cluster head. So, the measurement matrix can be divided into many sub-matrices; each sub-matrix corresponds to a cluster. $\phi^{H_{i}}$ represents the $i^{\text {th }}$ sub-matrix, $\mathrm{CH}_{i}$ represents its cluster head, and $x^{H_{i}}$ represents data vector of this cluster. $\mathrm{CH}_{i}$ can calculate the measurement values $\phi^{H_{i}} x^{H_{i}}$ of received data $x^{H_{i}}$ based on its sub-matrix. When $\mathrm{CH}_{i}$ generates its $\mathrm{M}_{i}$ predicted values, it forwards data to the sink node along the backbone tree which connects clustered heads to the sink node.

Assume that all of the nodes are divided into four clusters (because the 5 or 6 or 7 or 8 or other clusters are the same as that of four clusters, we select four clusters as an example), which are connected through a

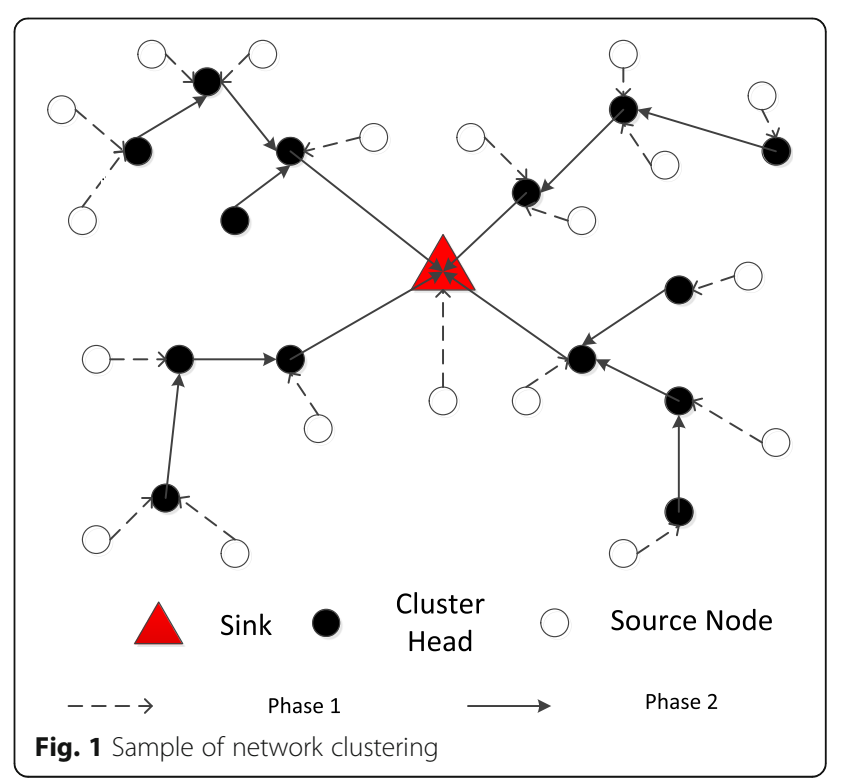


backbone aggregation tree. Data vector $x$ can be represented by $\left[\begin{array}{llll}x^{H_{1}} & x^{H_{2}} & x^{H_{3}} & x^{H_{4}}\end{array}\right]^{T}$. Matrix $\phi$ can be represented by $\left[\begin{array}{llll}\phi^{H_{1}} & \phi^{H_{2}} & \phi^{H_{3}} & \phi^{H_{4}}\end{array}\right]$. Generally, those assumptions mentioned in this paper are realistic, and their implications tell us that the truth is from the real scenarios of the applications, and the results can be tested the cases of the applications.

$$
y=\phi x=\left[\begin{array}{llll}
\phi^{H_{1}} & \phi^{H_{2}} & \phi^{H_{3}} & \phi^{H_{4}}
\end{array}\right]\left(\begin{array}{c}
x^{H_{1}} \\
x^{H_{2}} \\
x^{H_{3}} \\
x^{H_{4}}
\end{array}\right)=\sum_{i=1}^{4} \phi^{H_{i}} x^{H_{i}}
$$

As shown in Formula (2), the predicted coefficient of measurement matrix is the sum of all the measured coefficients in the cluster. Therefore, in each round, the cluster head generates predicted coefficients; all cluster heads forward the received predicted coefficients to the sink node. When the sink node collected $M$ rounds predicted value, it can recover the original data.

We define the compressive ratio as $\rho=M / N$, which means that the ratio is between the measurement value $M$ in the process of compressive sensing and the length $N$ of collected signal. It describes the compression efficiency of the entire network.

We define the relative reconstruction error as $\varepsilon=\frac{\|d-d\|_{2}^{2}}{\|d\|_{2}^{2}}$, i.e., the ratio between the absolute error and the true value, where $d$ is the true distance value of a certain node $i$ and its cluster head node and $\stackrel{\wedge}{d}$ is the measurement distance value of a certain node $i$ and its cluster head node.

\section{Data aggregating method based on compressive sensing in WSNs}

Although compressive sensing technology can effectively reduce the energy consumption of each node in the network, it is directly related to the measurement value $M$ in compressive sensing. When the value of $M$ is large, the energy consumption of nodes remains high. To solve this problem, a novel hybrid compressive sensing data aggregating method is proposed, which mainly consists of four parts: network clustering, building the appropriate inter-cluster routing tree, compressive sensing data aggregating in clusters, and cluster head transmitting data to the sink node. How to construct the routing tree and evolve the process of compressive sensing in clusters is shown below.

\subsection{Network model}

We make the following assumptions in the network (generally, those assumptions are realistic, and their implications tell us that the truth is from the real scenarios of the applications):
1) $N$ nodes randomly distribute in a circular perception area (the radius is $L$ ); the sink node is at the center of the sensing area (as shown in Fig. 1).

2) The sink node has enough data space and the ability of process.

3) The initial energy and the transmission rate of each sensor node are the same.

4) Nodes can know its own location information using the relative locating technology.

Lemma 1: Suppose that nodes in the wireless sensor network are distributed randomly, data aggregating in the cluster uses sparse matrices. If the cluster head is at the center of this cluster, then nodes consume least energy for each measurement value aggregating process.

Proof Assume that the $j^{\text {th }}$ cluster consists of $m_{j}$ nodes; the sparse ratio of the measurement matrix in the process of compressive sensing is $s$. In each aggregating process, the average number $m_{j}^{\prime}$ of nodes which involves in the aggregation of measurement values is:

$$
m_{j}^{\prime}=\sum_{i=1}^{m_{j}} s \times 1=m_{j} s
$$

Obviously, only $m_{j}^{\prime}$ nodes need to forward their corresponding weights for each time. Therefore, cluster head node receives $m_{j}^{\prime}$ packets. So, at every measurement, the average energy consumption in the $j^{\text {th }}$ cluster is:

$$
\begin{aligned}
\bar{E}_{\text {intra }}^{j}=\sum_{i=1}^{m_{j}^{\prime}} E_{T x}^{i}\left(k, E\left(d_{i}\right)\right)+m_{j}^{\prime} E_{R x}(k) \\
=k \sum_{i=1}^{m_{j}^{\prime}}\left(E_{\text {ele }}+\varepsilon_{\text {amp }} E\left(d_{i}^{2}\right)\right)+m_{j}^{\prime} k E_{\text {ele }} \\
=2 m_{j}^{\prime} k E_{\text {ele }}+k \varepsilon_{\text {amp }} \sum_{i=1}^{m_{j}^{\prime}} E\left(d_{i}^{2}\right)
\end{aligned}
$$

where $E_{T x}^{i}\left(k, E\left(d_{i}\right)\right)$ represents the energy consumption consumed by the $i^{\text {th }}$ node when forwarding $k$ bit data to its cluster head. $E\left(d_{i}\right)$ represents the distance expectations from the $i^{\text {th }}$ node to its cluster head. As shown in the formula above, the average energy consumption is decided by $E\left(d_{i}^{2}\right)$. Suppose that the cluster is square and its side length is $b$ and the cluster head's coordinate $\left(x_{0}\right.$, $\left.y_{0}\right)$. We can use $f(x, y)$ to represent the probability density function of the distance between child nodes to the cluster head: 


$$
f(x, y)=\left\{\begin{array}{cc}
\frac{1}{b^{2}} & x \in\left(-\frac{b}{2}, \frac{b}{2}\right), y \in\left(-\frac{b}{2}, \frac{b}{2}\right) \\
0 & \text { other }
\end{array}\right.
$$

then

$$
\begin{aligned}
E\left(d_{i}^{2}\right)=E( & \left.\left(x-x_{0}\right)^{2}+\left(y-y_{0}\right)^{2}\right) \\
& =\int_{-\frac{b}{2}}^{\frac{b}{2}} \int_{-\frac{b}{2}}^{\frac{b}{2}} \frac{1}{b^{2}}\left(\left(x-x_{0}\right)^{2}+\left(y-y_{0}\right)^{2}\right) d x d y \\
& =\frac{b^{2}}{6}+\left(x_{0}^{2}+y_{0}^{2}\right) \geq \frac{b^{2}}{6}
\end{aligned}
$$

is true if and only if $x_{0}=y_{0}=0$, i.e., the cluster head node is at the center area of the cluster.

Assuming that the network is divided into $N_{c}$ non-overlapping clusters, that means $N_{c}$ nodes are selected as the cluster heads; the other nodes connect to the cluster head near to them.

We also assume that the node can adjust their own energy levels based on real transmission distance. Thus, the energy consumption from node $n_{i}$ to node $n_{j}$ is $P_{i j}=d_{i j}^{\alpha}$. The parameter $\alpha$ depends on the characteristics of the channel, which usually take between 2 and 4 as mentioned by References [13, 14]. Here, we choose $\alpha=2$, which is realistic for a typical WSN deployment [13-16]. Eventually, we use the normalized reconstruction error as the CS signal reconstruction error.

\subsection{Establishment of inter-cluster routing tree}

Hops are forwarded from current cluster head to other cluster head $(\mathrm{NoH})$, i.e., the node determines the value based on its own communication radius and the distribution of cluster heads in the network.

Lemma 2: Suppose that cluster heads forward measurement values along the inter-cluster multi-hop shortest routing tree, so the energy consumption of inter-cluster will reach to the minimum value.

Proof The cluster head will get $h-1$ data packets at each time collecting measurement values, and the definition of the energy consumption of inter-cluster is as follows:

$$
\begin{aligned}
E_{\text {inter }}=\sum_{i=1}^{h} & E_{T x}^{i}\left(k, d_{i}\right)+(h-1) E_{R x}(k) \\
= & k \sum_{i=1}^{h}\left(E_{\text {ele }}+\varepsilon_{\text {amp }} d_{i}^{2}\right)+(h-1) k E_{\text {ele }} \\
& =(2 h-1) k E_{\text {ele }}+k \varepsilon_{\text {amp }} \sum_{i=1}^{h} d_{i}^{2}
\end{aligned}
$$

where $d_{i}$ represents the transmission distance of the $i^{\text {th }}$ data packet. The formula above shows that if $h$ and $k$ are constant, the final result is decided by $\sum_{i=1}^{h} d_{i}^{2}$.

We propose an iterative algorithm to build distributed inter-cluster routing. Assuming that all cluster heads have the same transmission radius $(R)$. Within the communication radius, cluster heads can communicate with each other. All cluster heads broadcast the hops from themselves to the sink node to their neighbors. The $\mathrm{NoH}$ of cluster head which contains the sink node in their communication radius is set as 1 at the first time of iterating. At the next iteration, these cluster heads broadcast their $\mathrm{NoH}$ to their neighbors and set the $\mathrm{NoH}$ of those cluster head nodes without $\mathrm{NoH}$ to be 2. After a series of iterations, it keeps choosing routing path until no cluster head is left. The algorithm can be abbreviated as the following steps:

1 Do While(routing path no longer change)

$2 N o H(\operatorname{Sink})=0 ; i \in N_{c}$ cluster heads

3 Nei represents the set of neighbor nodes of cluster head $i$

4 If distance $[i, j]<R, \quad j \in R$

$5 \quad$ When $\mathrm{NoH}(\mathrm{j})=\min \{\mathrm{NoH}(\mathrm{Nei})\}$

$6 \quad$ The $i$-th cluster head connects with the $j$-th cluster head;

$7 \quad$ Set the $i$-th cluster head: $\mathrm{NoH}(i)=\mathrm{NoH}(j)+1$;

$8 \quad$ End If

9 End While

\subsection{Intra-cluster data aggregating based on compressive sensing}

After building inter-cluster routing tree, we use compressive sensing technology to collect data in clusters. Since the data correlation of intra-cluster node is relatively large, we can reduce the measurement values by using random space sparse matrix. In traditional data aggregation methods based on compressive sensing, the measurement matrix required for compressive sensing process is generated by the cluster head. When data is collected, the cluster head needs to forward both data and measurement matrix to the sink node. Because the random space sparse matrix can be directly generated by the sink node by using a sparse seed vector, each cluster head can generate its corresponding sub-matrix by using the seed vector provided by the sink node. The steps of the method are as follows: 
Step 1: The sink node forwards the seed vector $U\left(u_{i}\right),\{i=1,2, \ldots, N\}$ with sparse space $\triangle$ to every cluster head. Each cluster head determinating its position in the seed vector depends on its position on the backbone tree.

Step 2: Start from its position in the seed vector, the $i^{\text {th }}$ cluster head node traverses forward $N_{i}$ values depends on the number of its intra-cluster nodes $N_{i}$. Then, the cluster head gets its own new sparse seed vector and eventually generates its corresponding sub-matrix $M_{i} \times N_{i}$.

Step 3: Non-CH (cluster head, $\mathrm{CH}$ ) nodes forward their nodes to $\mathrm{CH}$; $\mathrm{CHs}$ calculate the received data as $M_{i}$ measurement values by using the formula $y_{i}=\phi_{i} x_{i}$.

Step 4: CHs forward measurement values to the sink node along the generated forwarding path.

Step 5: The sink node generates the whole measurement matrix based on the whole seed vector $U\left(u_{i}\right),\{i=1,2, \ldots, N\}$ and recovers the original data depends on received data $y$ $=\left[y_{1}, y_{2}, \ldots, y_{N c}\right]$ by using CS reconstruction algorithm.

\section{Analysis of energy consumption in WSN}

As stated in the above sections, non- $\mathrm{CH}$ nodes send their readings to their cluster heads. The energy consumption of intra-cluster defines as $P_{\text {intra-cluster. }}$ In the next step, the cluster heads get their corresponding measurement values $\left(y_{i}=\phi_{i} x_{i}\right)$ based on intra-cluster node data and then send measurement values to the sink node. The energy consumption of intra-cluster node represents as $P_{\text {toBS }}$, and total energy consumption is expressed as follows:

$$
P_{\text {total }}=\left(P_{\text {intra-cluster }}+P_{\text {toBS }}\right)
$$

\section{1) Analysis of $P_{\text {intra-cluster }}$}

We assume that WSN is divided into $N_{c}$ clusters evenly, each cluster has the same number of nodes $N / N_{c}$, including a cluster head and $N / N_{c}-1$ non- $\mathrm{CH}$ nodes. Then,

$$
P_{\text {intra-cluster }}=N_{c}\left(\frac{N}{N_{c}}-1\right) E\left[r^{\alpha}\right]
$$

where $r$ is a random variable, which corresponds to the distance between a common node and its cluster head; $\alpha$ is the path loss exponent. In this paper, we set it as 2, so we can calculate the expectation $E\left[r^{2}\right]$ :

$$
E\left[r^{2}\right]=\iint\left(x^{2}+y^{2}\right) \rho(x, y) d x d y=\iint r^{\prime 2} \rho\left(r^{\prime}, \theta\right) r^{\prime} d r^{\prime} d \theta
$$

where $\rho(r, \theta)$ represents the distribution of nodes. We also assume that each cluster is a circular area of $R=L / \sqrt{N_{c}}$ radius; the density of nodes in all clusters is distributed evenly. Therefore,

$$
E\left[r^{2}\right]=\frac{1}{\left(\pi L^{2} / N_{c}\right)} \int_{\theta=0}^{2 \pi} \int_{r^{\prime}=0}^{R} r^{\prime 3} d r^{\prime} d \theta=\frac{L^{2}}{2 N_{c}}
$$

Correspondingly,

$$
P_{\text {intra-cluster }}=\left(\frac{N}{N_{c}}-1\right) \frac{L^{2}}{2}
$$

2) Analysis of $P_{\text {toBs }}$

We define the energy consumption of inter-cluster transmission as follows:

$$
P_{\mathrm{toBS}}=\sum_{i=1}^{N_{c}} N o H(i) \times R^{2} \times M(i)
$$

where $M(i)$ is the number of measurement values of the $i^{\text {th }}$ cluster; $R^{2}$ is the energy consumption on each hop. In the case of the analysis, we assume that all cluster sizes are equal. According to the literature [18], the number of measurement values required for each cluster is linearly proportional to the number of nodes in each cluster. Therefore, Eq. (12) can be rewritten as follows:

$$
P_{\mathrm{toBS}}=R^{2} \times \frac{M}{N_{c}} \sum_{i=1}^{N_{c}} N o H(i)
$$

As aforementioned, $M$ represents the total number of measured values required in the network. $N_{c}$ is the number of clusters. Formula (14) can be rewritten as follows:

$$
P_{\text {toBS }}=\mathrm{NoH}_{\text {ave }} \times R^{2} \times M
$$

where $\mathrm{NoH}_{\text {ave }}$ is the average number of hops.

3) Analysis of communication radius of cluster head

Communication radius of cluster head is closely related to the network energy consumption. In each routing path, the number of hops is closely related to the communication radius. If $R$ is increased, the cluster head can forward data to more cluster heads, which means that the total number of hops will change with the communication radius $R$. In Figs. 2 and 3, we construct a network of 2000 nodes. The network is clustered by using a common method such as 


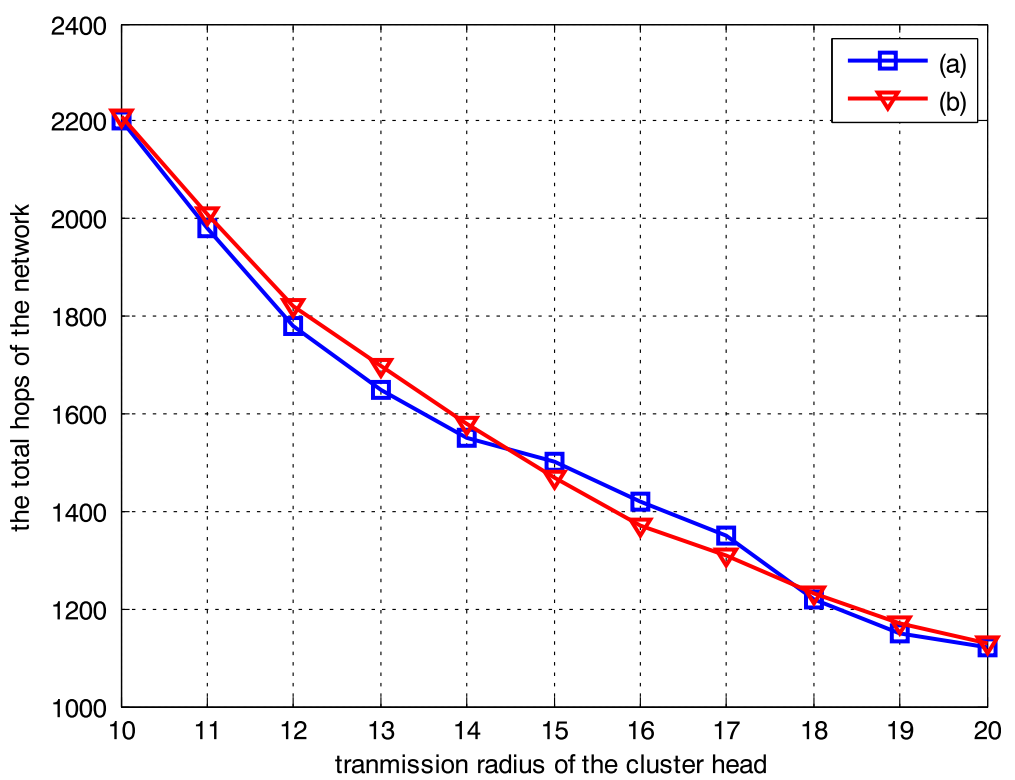

Fig. 2 Comparison of the total hops change in the network

K-means or LEACH. We change different communication radius $R=\{10,12,14,16,18,20\}$ in order to change the number of clusters in the network. As shown in Figs. 2 and 3, the total hops will change after increasing or decreasing of radius $R$ correspondingly. Generally, the units of the communication radius $R$ used to measure the quantities are specified meter as $m$ or the times of $m$, so we ignore the unit description for the following figures.

Figure 2 shows the comparison of the total hop change in the network when changing the communication range of the cluster head. Figure 3 shows the comparison of the total energy consumption of the network when changing the communication range of the cluster head. The figures above (a) uses K-means before data aggregating and (b) uses LEACH before data aggregating.

\section{Description of the algorithm}

Theorem 1 Assuming that wireless sensor network is clustering uniformly, the intra-cluster collect data by using compressive sensing technology, sparse matrix is

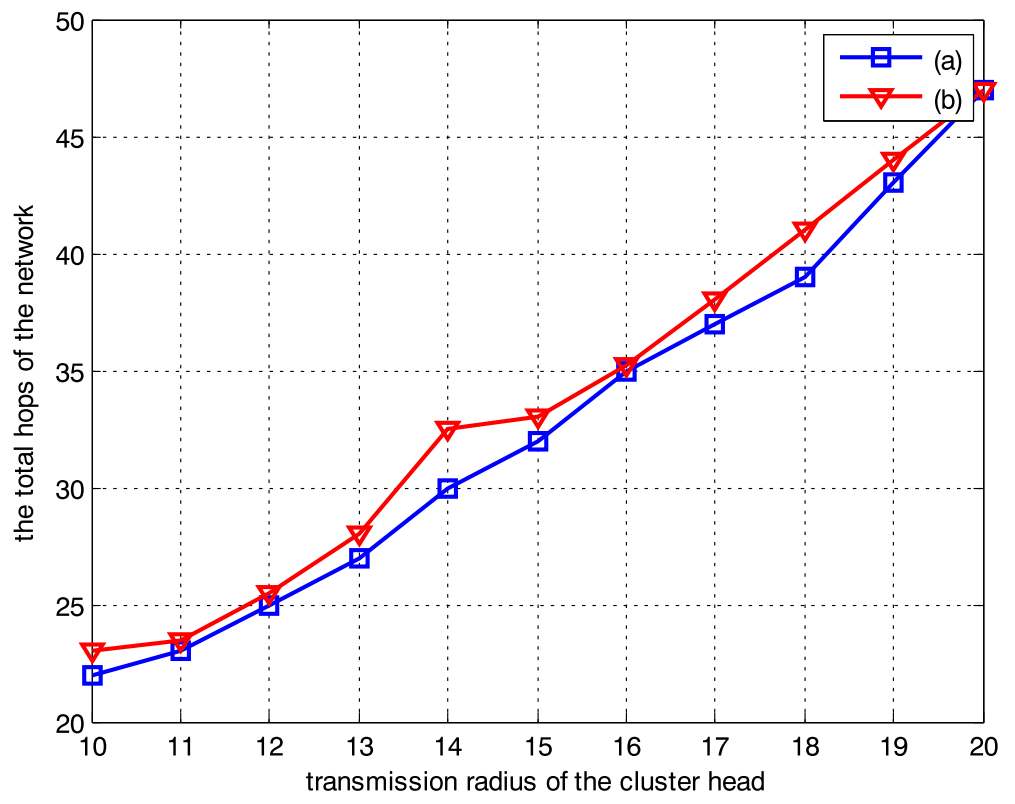

Fig. 3 Comparison of the total energy consumption of network 
selected as the measurement matrix, cluster head node is at the center of cluster, and inter-cluster forwards data along the shortest multi-hop routing tree. Then every time in the data aggregating, the total energy consumption of network is minimum.

Proof From the previous Lemma 1 and Lemma 2, the mean value of energy consumption in the wireless sensor network is as follows:

$$
\begin{aligned}
E_{\text {total }}(h)= & \sum_{i=1}^{h} \bar{E}_{\text {intra }}^{i}+E_{\text {inter }}=k E_{\text {ele }}\left(2+\frac{\varepsilon_{\text {amp }} b^{2}}{6}\right) \sum_{i=1}^{h} m_{i}^{\prime} \\
& +(2 h-1) k E_{\text {ele }}+k \varepsilon_{\text {amp }} \sum_{i=1}^{h} d_{i}^{2}
\end{aligned}
$$

where $m_{i}^{\prime}$ represents the average number of nodes within $i^{\text {th }}$ cluster the first time to participate in a single measurement. In the case of uniform clustering, $m_{i}^{\prime}=m_{j}^{\prime}$ and $d_{i}^{2}=d_{j}^{2}(i, j=1,2, \ldots, h, i \neq j)$ then $\sum_{i=1}^{h} m_{i}^{\prime}$ and $\sum_{i=1}^{h} d_{i}^{2}$ reach the minimum. So, $\bar{E}_{\text {total }}(h)$ reaches the minimum.

This section presents a kind of data aggregating algorithm based on hybrid compressive sensing, which is different from the traditional hybrid compressive sensing data aggregation. The measurement matrix required for every cluster is generated by seed vector provided by the sink node; because all of the intra-cluster nodes have the same calculation process, the entire network has balanced energy consumption. The complete algorithm is described as follows:

1) The network is clustered by using conventional clustering methods, such as LEACH and K-means.

2) The aforementioned method is used to construct the inter-cluster multi-hop shortest routing tree between cluster heads and the sink node. Each cluster head can get its own $\mathrm{NoH}$. As seen in Formula (13), if $M$ and $N_{c}$ are certain, the energy consumption of inter-cluster is only associated with $\mathrm{NoH}$.

3) The sink node generates a corresponding sparse seed vector $U\left(u_{i}\right),\{i=1,2, \ldots, N\}$ according to the number of nodes in the network and send it to each cluster head.

4) Each cluster head (assuming that $i^{\text {th }}$ cluster head) using the received seed vector generates its measurement matrix $M_{i} \times N_{i}$ according to its location and the number of nodes in it.

5) In the cluster, data is collected by using compressive sensing technology, then we can get $M$ measurement values of the corresponding cluster head.
6) Cluster heads forward $M$ measurement values to the sink node along the inter-cluster multi-hop shortest routing tree. Based on Theorem 1, the total energy consumption of network during the data acquisition is minimum, so as to achieve the best performance; otherwise, we use machine learning approach to reconstruct signal and then ensure that the total energy consumption is minimum. Detailed machine learning approach can be found in our relative research works [7-11], because of the length limit of the paper, we ignore the detailed description.

7) Since the measurement matrix used in each cluster is generated by the partial seed sparse vector $U\left(u_{i}\right),\{i=1,2, \ldots, N\}$, so the sink node may also generate a total block matrix as the recovery matrix. The sink node recovers the original data by using corresponding reconstruction algorithm.

Because the random space sparse matrix can be dynamically generated by a series of seed vectors, the measurement matrix required for the whole network can be determined by the sink node. On one hand, compared with the Gaussian random matrix, it reduces the number of independent variables; on the other hand, it avoids the problem that nodes cannot save the dynamic measurement matrix while routing path changes in the process of conventional hybrid compressive sensing.

\section{Results and discussions}

This section provides some simulations and evaluations of this proposed data aggregating method.

\subsection{Performance of data aggregating based on random space sparse compressive sensing}

We always assess the performance of methods by using the amount of data packet transmission collected by nodes in the network; the space here is $\Delta=2$. We compare six schemes: (a) K-means clustering scheme based on random space sparse measurement matrix, (b) LEACH clustering scheme based on random space sparse measurement matrix, (c) K-means clustering scheme based on Gaussian measurement matrix, (d) LEACH clustering scheme based on Gaussian measurement matrix, (e) K-means clustering scheme without compressive sensing, and (f) LEACH clustering scheme without compressive sensing. The number of nodes is increased from 500 to 1500 , the transmission radius nodes is 10 , and the compressive ratio is $\rho=M / N$.

Figure 4 shows the comparison of data packet transmission of various programs when the compressive ratio is $\rho=0.2$. Figure 5 shows the comparison of data packet transmission of various programs when the compressive ratio is $\rho=0.1$. The two values of the compressive ratio $\rho$ are used, which are $\rho=0.1$ and $\rho=0.2$; what value of $\rho$ 


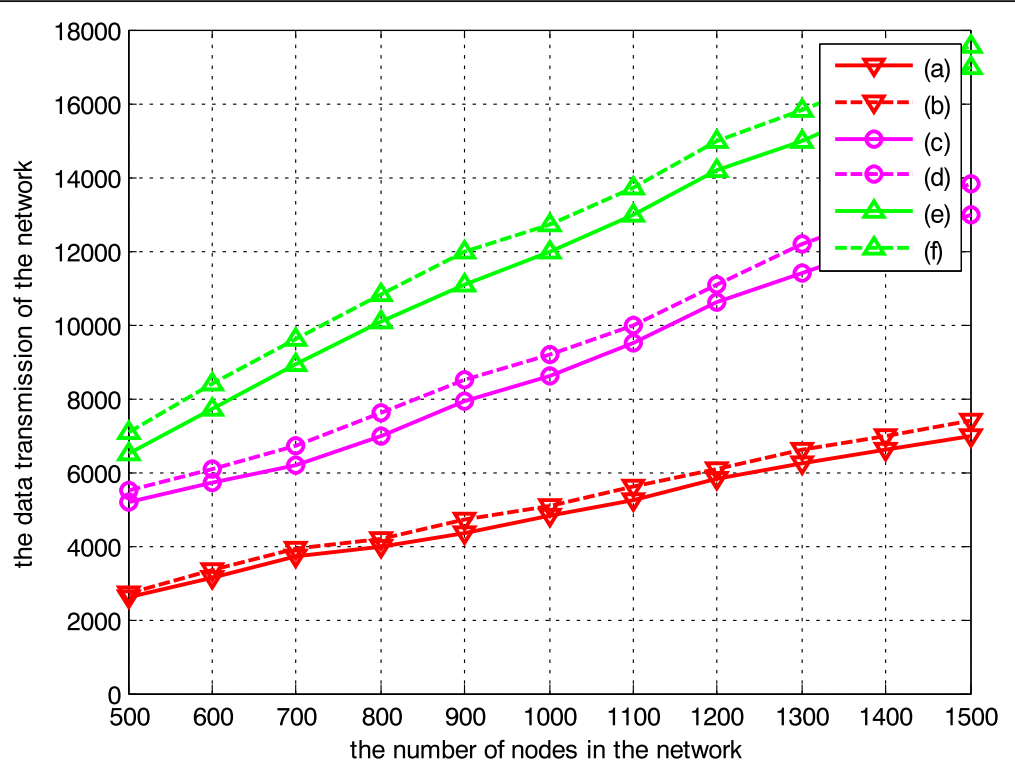

Fig. 4 Comparison of data packet transmission of various programs

would be a realistic one will be based on the requirements of the realistic applications, if the high ratio is needed, then selecting high ratio, such as $\rho=0.2$, which is related to the crucial importance and know the importance of the results.

Figure 6 shows the comparison of the tendency of the network lifecycle changes with the number of nodes when the compressive ratio is $\rho=0.1$. Figure 7 shows the comparison of the tendency of the network lifecycle changes with the number of nodes when the compressive ratio is $\rho=0.2$. It can be seen from the figures that the use of compressive sensing obviously prolongs the network's lifecycle, while compared to the Gaussian random matrix, random space sparse matrix collects less data packets, thereby further increases the number of rounds of the network.

\subsection{Simulation and analysis of energy consumption in network}

We also deploy 2000 nodes, and $L$ is 100 . Firstly, the network is clustered by K-means or LEACH, then we get $N_{c}$ clusters. We use our CS data aggregating method and calculate the energy consumption of the entire network. The sink node is set at the center of sensing field. Given

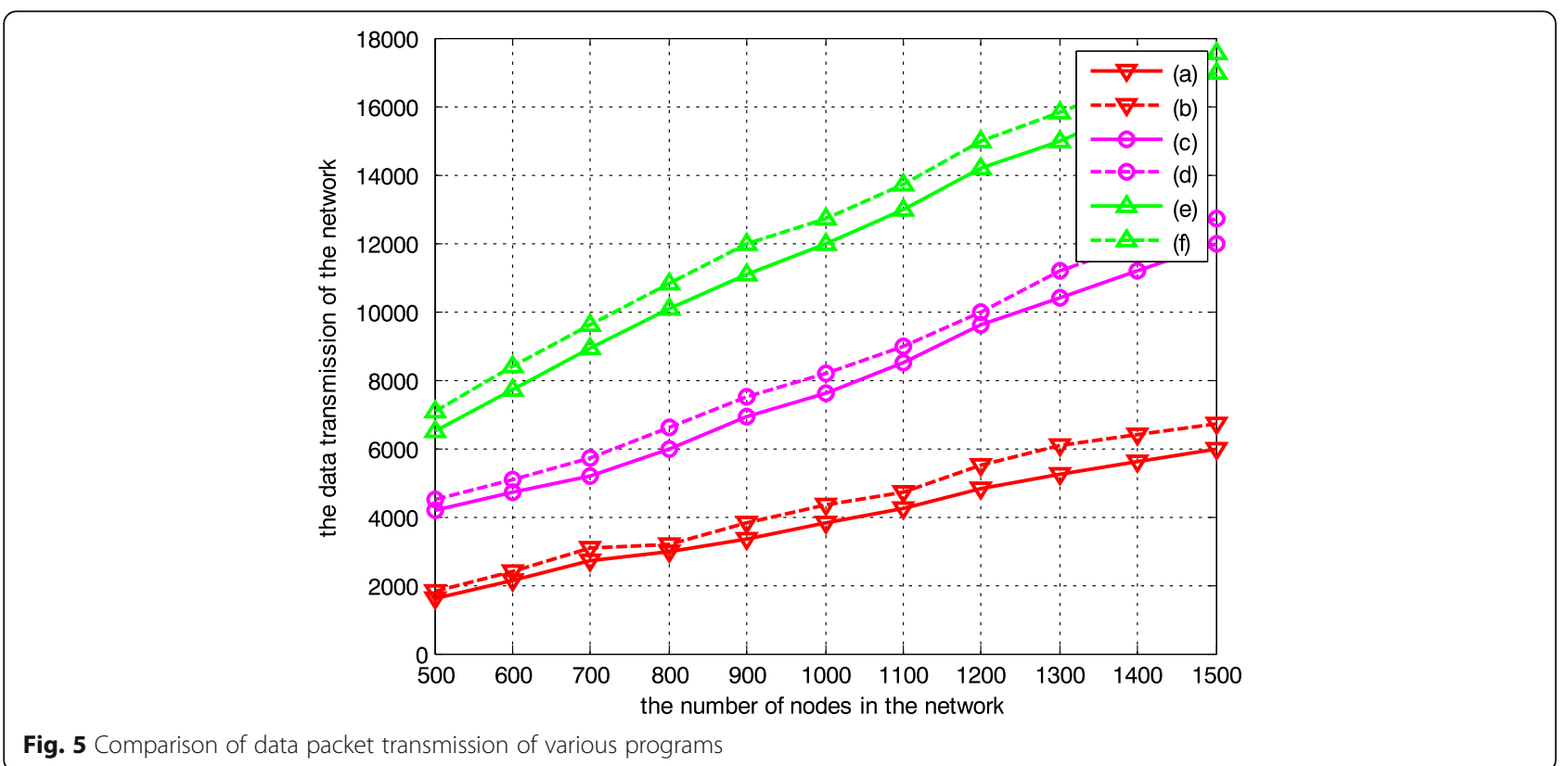




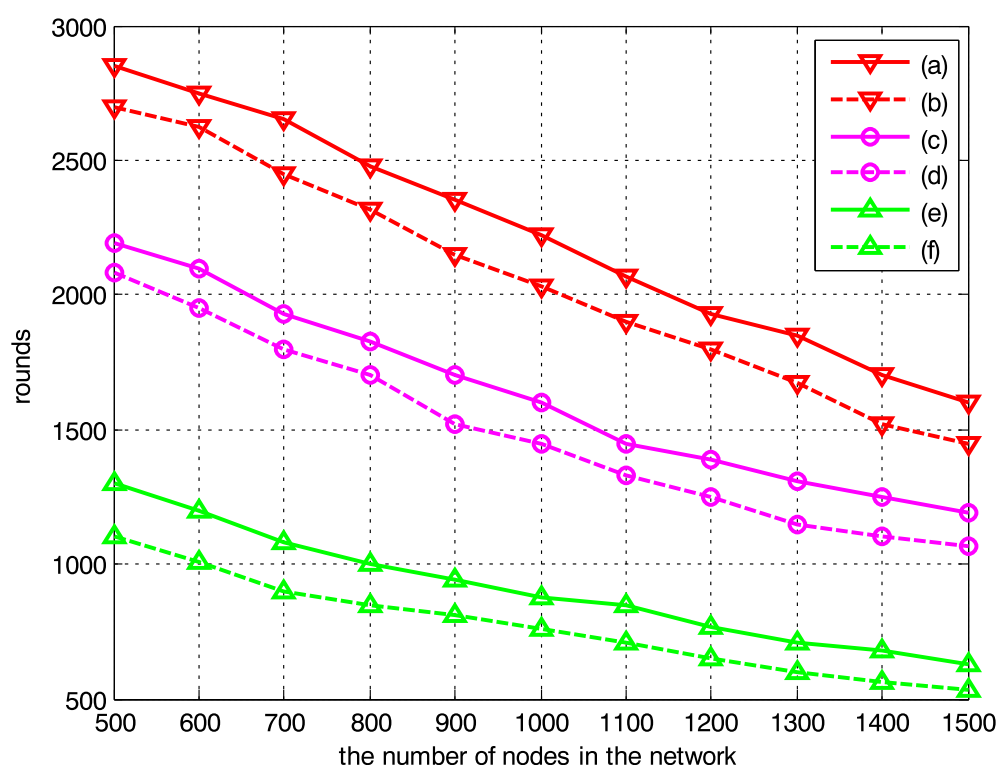

Fig. 6 Comparison of tendency of the network lifecycle changes with the number of nodes

the number of measurements $M=500$, in order to meet the target error 0.1, we change the number of cluster head of the network by changing the transmission radius of nodes. We use the transmission radius $R=[50,30,25$, $22,18,14,11]$ to represent the number of the cluster head $N_{c}=[10,50,100,200,300,400,500]$.

First, the energy consumption of intra-cluster is simulated. We select random space sparse matrix and Gaussian matrix to do the comparison, and we also choose the different random space $\Delta$. As shown in Figs. 8 and 9 ((a) represents as the program using K-means and random space sparse matrix, (b) as the program using LEACH and random space sparse matrix, (c) as the program using K-means and Gaussian random matrix, and (d) as the program using LEACH and Gaussian random matrix), they represent the total energy consumption of intra-cluster including cluster head. If the number of cluster increases, then the energy consumption of intra-cluster decreases. At this time, the transmission of data packet of inter-cluster consumes much energy. As

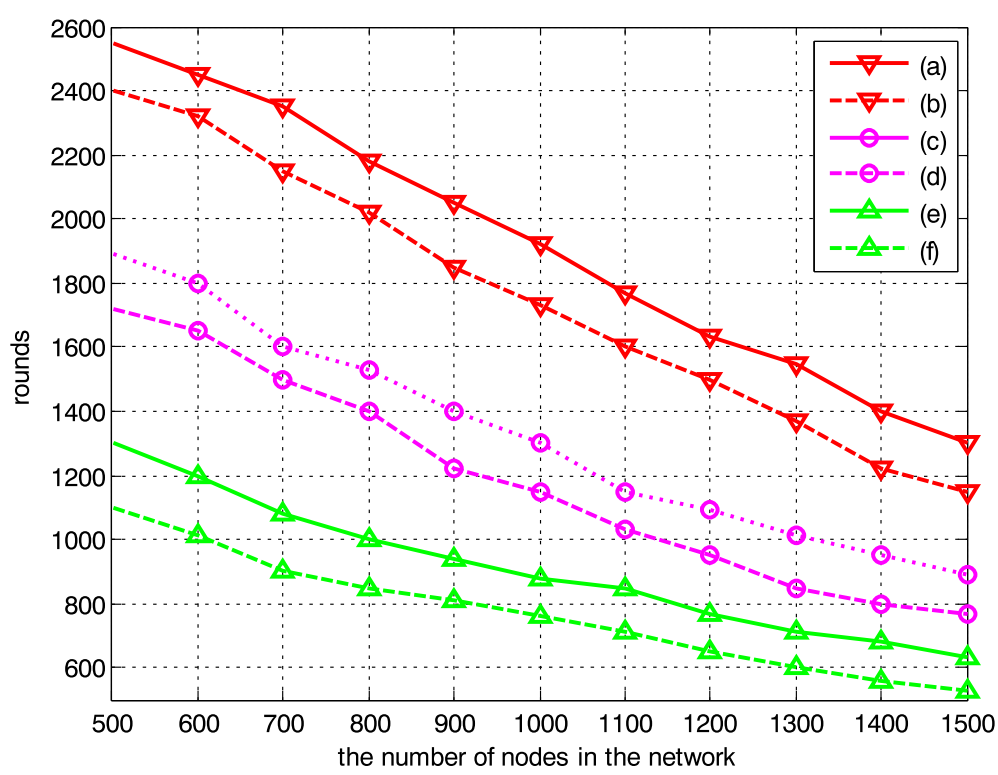

Fig. 7 Comparison of tendency of the network lifecycle changes with the number of nodes 


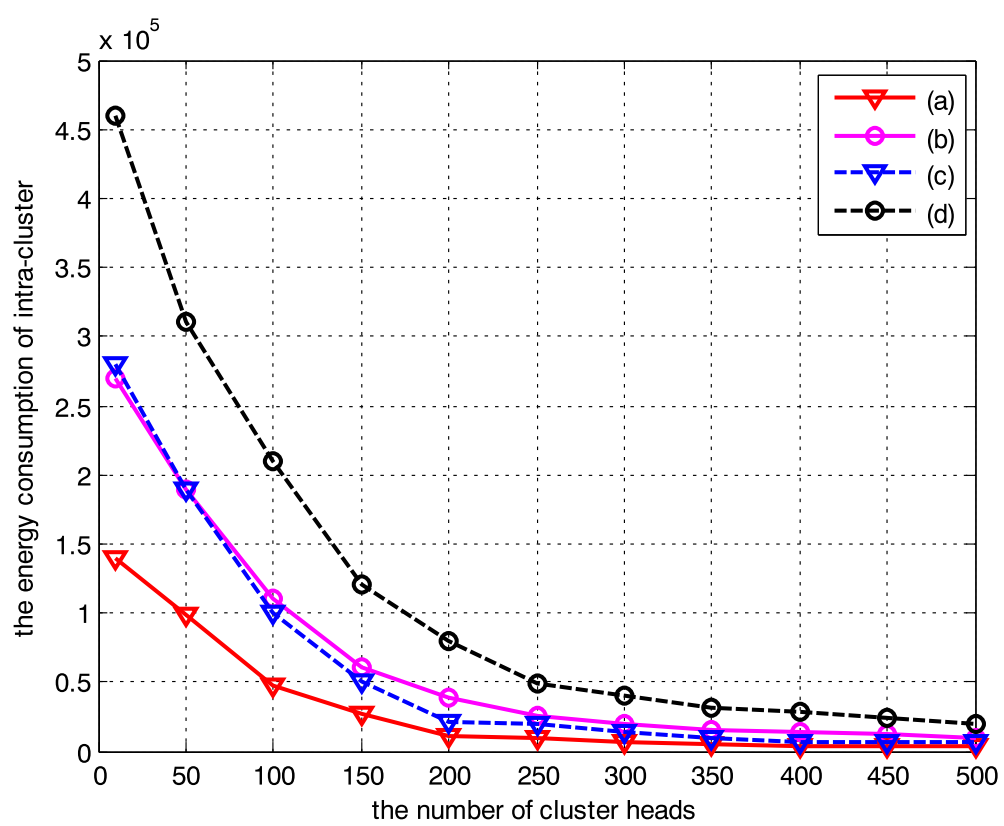

Fig. 8 Comparison of change of the energy consumption of intra-cluster

can be seen from the figures, the random space sparse matrix consumes less than the Gaussian matrix due to a large number of zero element.

With the increase of the number of cluster heads, we represent (a) as the program using K-means and random space sparse matrix with $\Delta=2$, (b) as the program using $\mathrm{LEACH}$ and random space sparse matrix with $\Delta=2$, (c) as the program using K-means and Gaussian random matrix, and (d) as the program using $\mathrm{LEACH}$ and Gaussian random matrix.

In addition, with the increase of the number of clusters, we represent (a) as the program using $\mathrm{K}$-means and random space sparse matrix with $\Delta=4$, (b) as the program using LEACH and random space sparse matrix

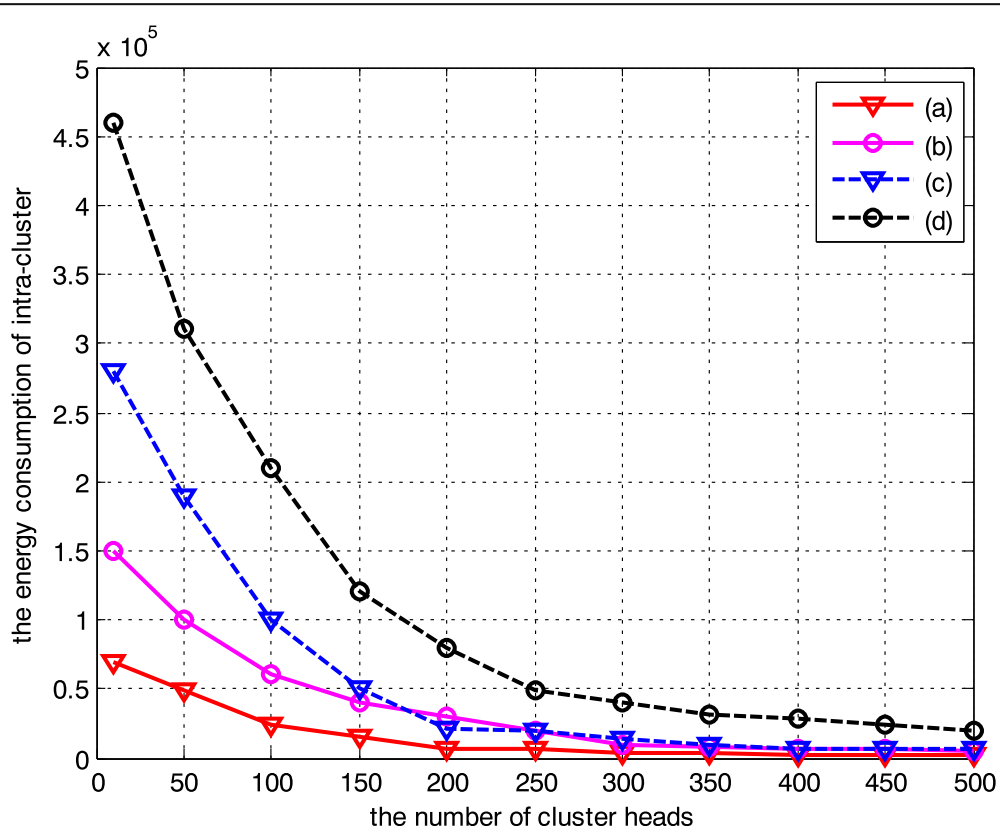

Fig. 9 Comparison of change of the energy consumption of intra-cluster 


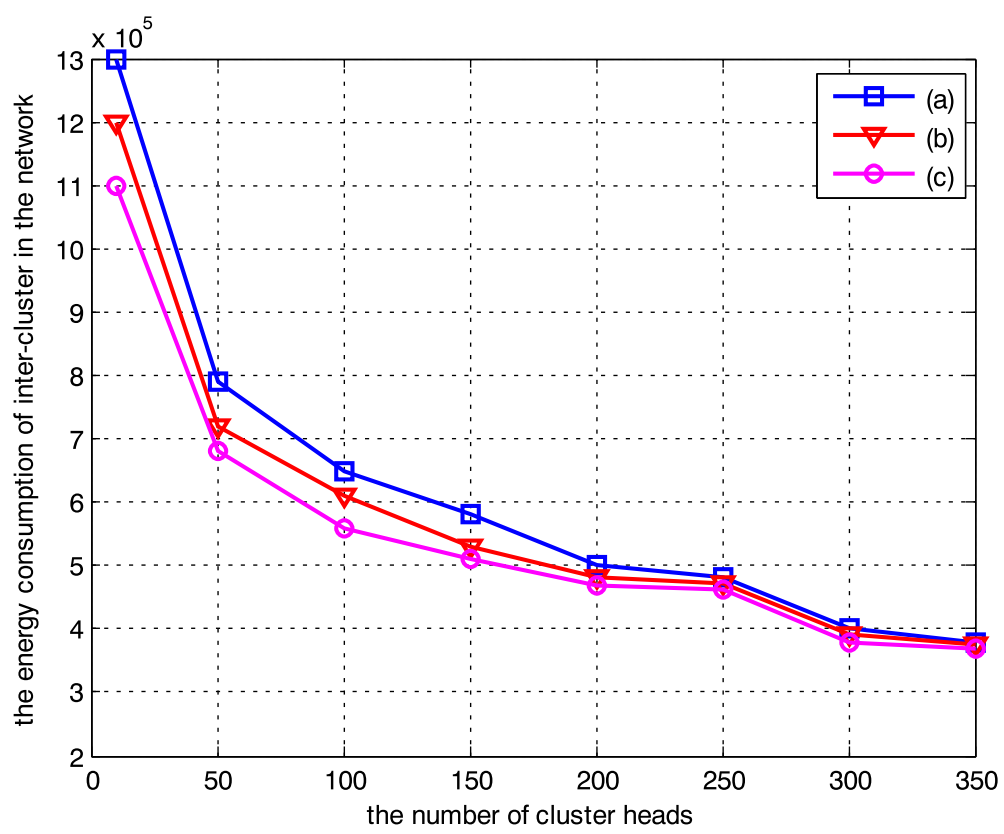

Fig. 10 Comparison of change of the energy consumption of inter-cluster

with $\Delta=4$, (c) as the program using K-means and Gaussian random matrix, and (d) as the program using LEACH and Gaussian random matrix.

Figure 10 shows that the entire energy consumption of inter-cluster is decreased with the increasing of the number of clusters. We represent (a) as uniform clustering, (b) as $\mathrm{LEACH}$, and (c) as K-means.
Figure 11 shows the trend of the total energy consumption of the network. It can be seen from the figure that the use of inter-cluster multi-hop routing significantly reduces the total energy consumption of the network when there are too many clusters. We represent (a) as the use of inter-cluster multi-hop routing and K-means, (b) as the use of inter-cluster multi-hop

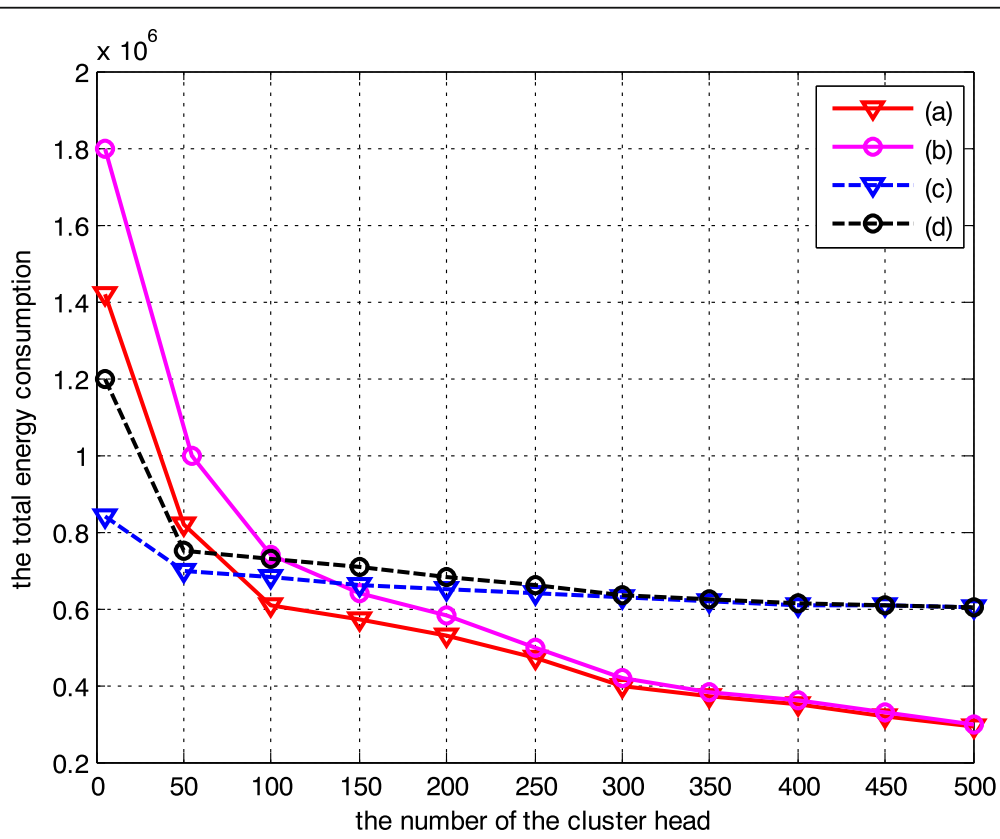

Fig. 11 Comparison of the total energy consumption of network 


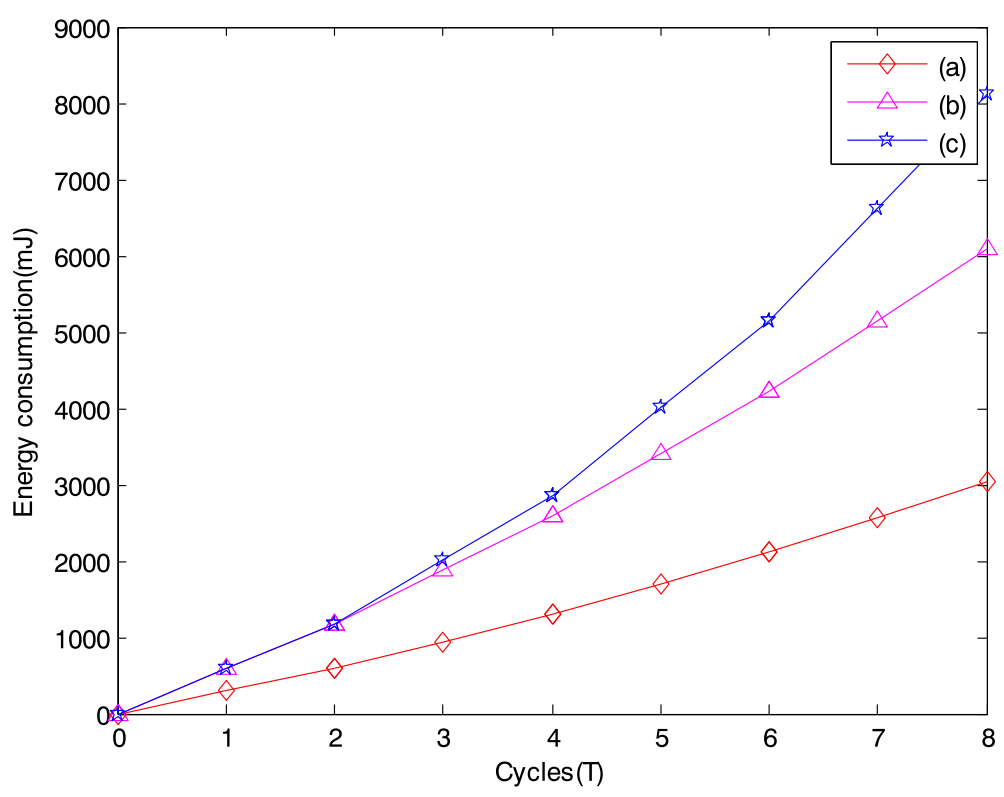

Fig. 12 Comparison of the energy consumption of network

routing and $\mathrm{LEACH}$, (c) as the only use of K-means, and (d) as the only use of LEACH.

In addition, we do the comparison experiments on the total consumption of network of WSN with the relative methods [19-27]. When we consider the total consumption of network, an abnormal situation occurs (in Fig. 12, the abnormal situation regarding new nodes added to the network in the actual applications because of the dynamic change of the network topology, such as some nodes go into the relative clusters or some nodes leave the relative clusters, the implication is that the WSN is self-organized based on the requirements of the relative applications), which has added new nodes in the data collection process. In order to consider the worst case, we assume that new nodes are added at the front end of the network. Fifty new nodes are joined in the network every 2 cycles. From the results of Fig. 12 (in Fig. 12, we represent (c) as the method of Reference [19], we

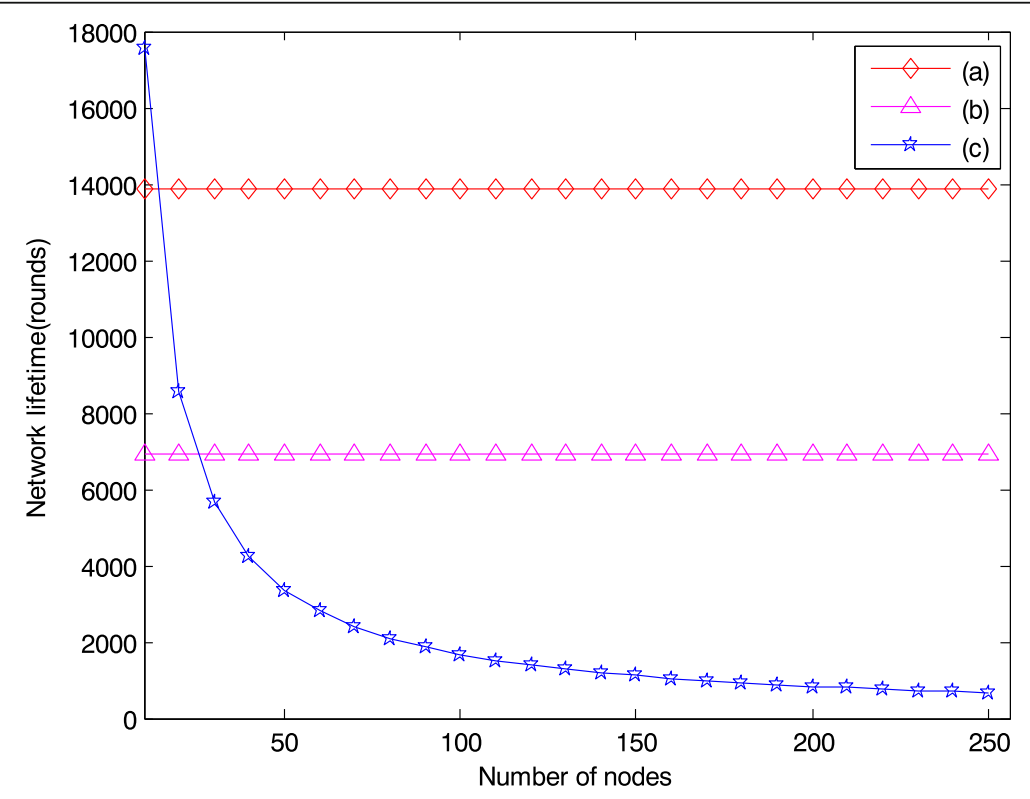

Fig. 13 Comparison of lifetime of network under different data collection methods 


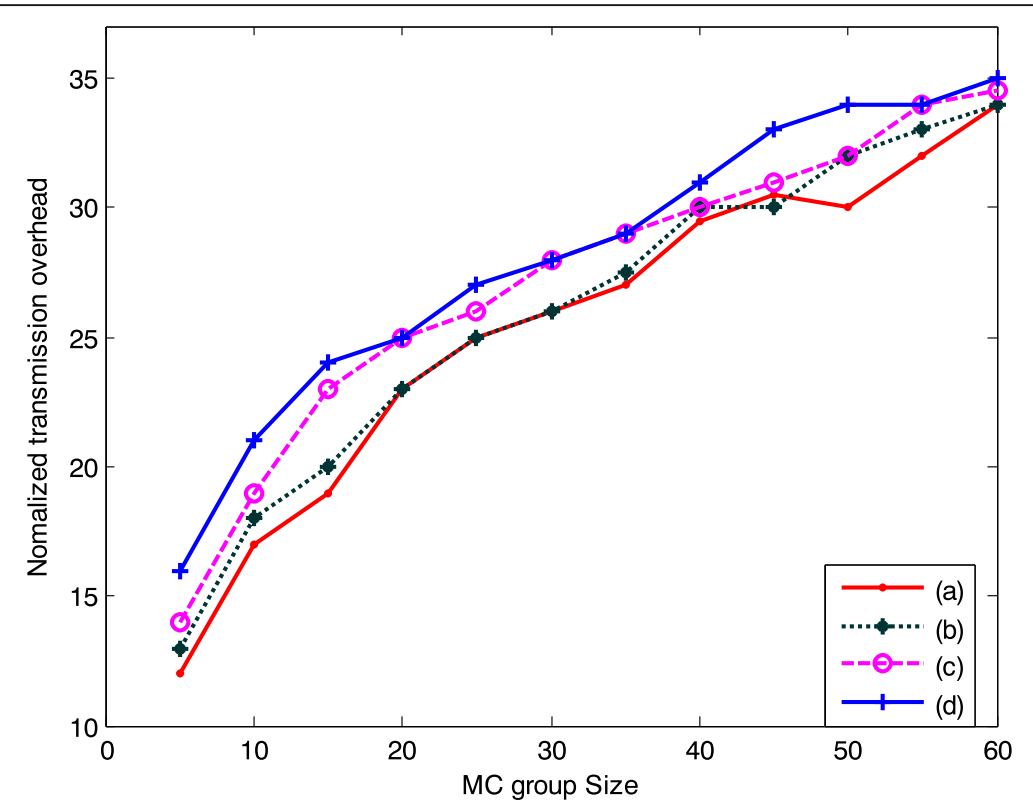

Fig. 14 Overhead comparison of normalized transmission

represent (b) as the method of Reference [20], we represent (a) as the method of this paper), we can see that our method reduces the total energy consumption of network than that of other methods regardless of intra-cluster or inter-cluster.

Figure 13 shows the comparison of lifetime of network under different data collection methods. In Fig. 13, we represent (c) as the method of Reference [21], we represent (b) as the method of Reference [23], and we represent (a) as the method of this paper. From the results of Fig. 13, we can see that our method prolongs the lifetime of network than that of other methods.

The algorithm can be described as intra-cluster method based on existing methods and the inter-cluster aggregation based on minimum consumption. The common problem in clustering networks which is the energy balancing during the head selection is well considered by the machine learning process.

The WSNs will inevitably use clustering when the node number is large. It is not a fair comparison between the cluster and non-cluster structure in large-scale networks, so we adopt the overhead of normalized network transmission based on the relative weight.

In order to compare our work with other clustering methods and including the machine learning process, the cost of the algorithm (the bandwidth, energy consumption caused by the extra communication) is considered on the performance analysis as Fig. 14 ((a) as the method of this paper, (b) as the method of Reference [23], (c) as the method of Reference [21], and (d) as the method of Reference [24]).
From Fig. 14, we can see that optimized compressive sensing data collection program reduces the overhead of normalized network transmission than the un-optimized program.

\section{Conclusions}

A kind of effective data aggregating method based on compressive sensing in WSN is proposed. The method can effectively reduce the energy consumption of the network. The sink node forwards sparse seed to cluster heads. Within a cluster, the cluster head generates its required measurement matrix according to the received sparse seed and then produces the corresponding measurement values by using random space sparse compressive sensing. Cluster heads forward measurement values to the sink node along the inter-cluster multi-hop routing tree from one cluster to another. The sink node reconstructs the original signal by using the corresponding compressive sensing reconstruction algorithm. We analyze the energy consumption of the algorithm in the network, the relationship between the size of cluster head and the energy consumption of inter-cluster, and the relationship between the size of cluster head and the energy consumption of network. The experimental results show that this method can effectively reduce the energy consumption of the network.

\section{Acknowledgements}

This research work is supported by the National Natural Science Foundation of China (Grant No. 61571328), Tianjin Key Natural Science Foundation (No.13JCZDJC34600), CSC Foundation (No. 201308120010), Major projects of science and technology in Tianjin (No.15ZXDSGX 00050), training plan of Tianjin University Innovation Team (No.TD12-5016), major projects of science and technology for their services in Tianjin (No.16ZXFWGX00010, 
No.17YFZCGX00360), the Key Subject Foundation of Tianjin (15JCYBJC46500), and training plan of Tianjin 131 Innovation Talent Team (No.TD2015-23).

\section{Funding}

The work is partially supported by the following funding: training plan of Tianjin University Innovation Team (No.13-5025).

\section{Availability of data and materials}

The data will not be shared due to confidentiality matters.

\section{Authors' contributions}

D-gZ designed the algorithm. TZ wrote this paper. JZ did the experimental tests. YD optimized the algorithm and experiments. $X$-dZ checked the whole paper and figures. All authors read and approved the final manuscript.

\section{Authors' information}

De-gan Zhang, Ph.D., graduated from the Northeastern University, China. Now, he is a visiting professor of School of Electronic and Information Engineering, University of Sydney, Sydney, NSW 2006, Australia; professor of Tianjin Key Lab of Intelligent Computing and Novel software Technology, Key Lab of Computer Vision and System, Ministry of Education, Tianjin University of Technology, Tianjin, 300384, China. His research interest includes image processing, service computing, etc.

Ting Zhang, Ph.D., is a member (M) of IEEE in 2012. Now, she is a researcher at Tianjin University of Technology, Tianjin, 300384, China. Her research interest includes WSN, mobile computing, etc.

Jie Zhang (Beijing No.20 High School, Xiaoyingxilu, Haidian District, Beijing 100085, China). His research interest includes image processing, CRN, WSN, and IOT.

Yue Dong, Ph.D., is a researcher at Tianjin University of Technology, Tianjin, 300384, China. Her research interest includes WSN, etc.

Xiao-dan Zhang, Ph.D., is a member (M) of IEEE in 2012. Now, she is a researcher at Institute of Scientific and Technical Information of China, Beijing, 100038, China. Her research interest includes WSN, mobile computing, etc.

\section{Ethics approval and consent to participate}

Not applicable.

\section{Competing interests}

The authors declare that they have no competing interests.

\section{Publisher's Note}

Springer Nature remains neutral with regard to jurisdictional claims in published maps and institutional affiliations.

\section{Author details}

'Key Laboratory of Computer Vision and System, Tianjin University of Technology, Ministry of Education, Tianjin 300384, China. ${ }^{2}$ Tianjin Key Lab of Intelligent Computing and Novel Software Technology, Tianjin University of Technology, Tianjin, China. ${ }^{3}$ Beijing No. 20 High School, Xiaoyingxilu, Haidian District, Beijing 100085, China. ${ }^{4}$ Institute of Scientific and Technical Information of China, Beijing 100038, China.

\section{Received: 22 February 2018 Accepted: 5 June 2018}

\section{Published online: 19 June 2018}

\section{References}

1. C Luo, W F, J Sun, Efficient measurement generation and pervasive sparsity for compressive data gathering. IEEE Trans. Wirel. Commun. 9(12), 37283738 (2011)

2. DG Zhang, G Li, K Zheng, An energy-balanced routing method based on forward-aware factor for wireless sensor network. IEEE Trans Ind Inf 10(1), 766-773 (2014)

3. YY Xiao, Time-ordered collaborative filtering for news recommendation. China Commun 12(12), 53-62 (2015)

4. DG Zhang, X Wang, XD Song, A novel approach to mapped correlation of ID for RFID anti-collision. IEEE Trans. Serv. Comput. 7(4), 741-748 (2014)

5. XD Zhang, Design and implementation of embedded un-interruptible power supply system (EUPSS) for web-based mobile application. Enterp Inf Syst 6(4), 473-489 (2012)
6. SYi, J Heo, Y Cho, PEACH: power-efficient and adaptive clustering hierarchy protocol for wireless sensor networks. Comput. Commun. 30(14-15), 28422852 (2007)

7. K Zheng, Novel quick start (QS) method for optimization of TCP. Wirel. Netw 22(1), 211-222 (2016)

8. K Zheng, T Zhang, A novel multicast routing method with minimum transmission for WSN of cloud computing service. Soft. Comput. 19(7), 1817-1827 (2015)

9. HL Niu, Novel PEECR-based clustering routing approach. Soft. Comput. 21(24), 7313-7323 (2017)

10. DG Zhang, A new approach and system for attentive mobile learning based on seamless migration. Appl. Intell 36(1), 75-89 (2012)

11. YN Zhu, A new constructing approach for a weighted topology of wireless sensor networks based on local-world theory for the Internet of Things (IOT). Comput Math Appl 64(5), 1044-1055 (2012)

12. XJ Kang, A novel image de-noising method based on spherical coordinates system. EURASIP J Adv Sig Process 2012(110), 1-10 (2012). https://doi.org/ 10.1186/1687-6180-2012-110

13. J Haupt, WU Bajwa, Toeplitz compressed sensing matrices with applications to sparse channel estimation. IEEE Trans. Inf. Theory 56(11), 5862-5875 (2010)

14. C Zhang, HR Yang, Compressive sensing based on deterministic sparse Toeplitz measurement matrices with random pitch. Acta Automat. Sin. 38(8), 1362-1369 (2012)

15. YP Liang, A kind of novel method of service-aware computing for uncertain mobile applications. Math Comput Model 57(3-4), 344-356 (2013)

16. S Zhou, New mixed adaptive detection algorithm for moving target with big data. J Vibroengineering 18(7), 4705-4719 (2016)

17. R Devore, Deterministic constructions of compressed sensing matrices. J. Complex. 23(4), 918-925 (2007)

18. R Calderbank, S Howard, Construction of a large class of deterministic sensing matrices that satisfy a statistical isometry property. IEEE J Sel Top Sig Proces 4(2), 358-374 (2009)

19. B Malathi, Data collection based hybrid compressive sensing in wireless sensor networks. Int J Adv Inf Sci Technol (IJAIST) 4(2), 2319-2682 (2015)

20. GK Nigam, Effective compressive sensing for clustering in wireless sensor networks. Ind J Sci Technol 9(38), 0974-5645 (2016)

21. KA Shabna, Cluster method using hybrid compressive sensing for sensor network. Int J Modern Trends Eng Res (IJMTER) 2(5), 2349-9745 (2015)

22. M Kumar, $\mathrm{S}$ Verma, Clustering approach to data aggregation in wireless sensor networks. 16th IEEE Int Conference Netw 1(1), 125-135 (2008)

23. A Rajalakshmi, T Mohanraj, Efficient data transmission in wireless sensor networks using hybrid compressive sensing. J Recent Res Eng Technol 2(3), 2349-2252 (2015)

24. P Sukumar, B Sowmya, Effective hybrid compressive sensors using wireless networks in clustering methods. Int I Comput Sci Trends Technol (IJCST) 4(2), 110-119 (2016)

25. B Ameena, M Biradar, The hybrid compressive sensing data collection method in cluster structure for efficient data transmission in WSN. Int J Sci Res (IJSR) 4(6), 210-219 (2015)

26. NV Deshmukh, AV Deorankar, Consuming less energy in hybrid compressive sensed WSN. Natl Conference Adv Computing, Commun Netw 1(1), 30-40 (2016)

27. X Wang, XD Song, New medical image fusion approach with coding based on SCD in wireless sensor network. J Electr Eng Technol 10(6), 2384-2392 (2015)

28. $\mathrm{Z} \mathrm{Ma}$, Shadow detection of moving objects based on multisource information in Internet of Things. J Exp Theor Artif Intell 29(3), 649-661 (2017)

29. WB Li, Novel fusion computing method for bio-medical image of WSN based on spherical coordinate. J Vibroengineering 18(1), 522-538 (2016)

30. XD Song, X Wang, New agent-based proactive migration method and system for big data environment (BDE). Eng. Comput. 32(8), 2443-2466 (2015)

31. WB Li, Novel ID-based anti-collision approach for RFID. Enterp Inf Syst 10(7), 771-789 (2016)

32. DL Donoho, Compressed sensing. IEEE Trans Inform Theory 52(4), 12891306 (2006)

33. S Zhou, New Dv-distance method based on path for wireless sensor network. Intell Autom Soft Comput 23(2), 219-225 (2017)

34. X Wang, XD Song, New clustering routing method based on PECE for WSN. EURASIP J. Wirel. Commun. Netw. 2015(162), 1-13 (2015). https://doi.org/10. 1186/ s13638-015-0399-x 
35. S Liu, T Zhang, Novel unequal clustering routing protocol considering energy balancing based on network partition \& distance for mobile education. J. Netw. Comput. Appl. 88(15), 1-9 (2017). https://doi.org/10. 1016/j.jnca.2017.03.025

36. X Zhang, X Zhang, G C, A micro-artificial bee colony based multicast routing in vehicular ad hoc networks. Ad Hoc Netw. 58(4), 213-221 (2017)

37. KS He, YQ Li, CC Yin, A novel compressed sensing-based non-orthogonal multiple access scheme for massive MTC in $5 \mathrm{G}$ systems. EURASIP J. Wirel. Commun. Netw. 2018(81), 1-12 (2018)

38. JF Wan, B Zeng, A scalable and quick-response software defined vehicular network assisted by mobile-edge computing. IEEE Commun. Mag. 55(7), 94-100 (2017)

39. DY Jia, High-efficiency urban-traffic management in context-aware computing and 5G communication. IEEECommunications Mag 55(1), 34-40 (2017)

40. QR Wang, P Deng, A survey on position-based routing for vehicular ad hoc networks. Telecommun. Syst. 62(1), 15-30 (2016)

41. S Zhou, YM Tang, A low duty cycle efficient MAC protocol based on selfadaption and predictive strategy. Mob Netw Appl 2 (2017). https://doi.org/ 10.1007/s11036-017-0878-x

42. Z Ma, A novel compressive sensing method based on SVD sparse random measurement matrix in wireless sensor network. Eng. Comput. 33(8), 24482462 (2016)

43. H LNiu, Novel positioning service computing method for WSN. Wirel. Pers. Commun. 92(4), 1747-1769 (2017)

44. X Wang, A kind of novel VPF-based energy-balanced routing strategy for wireless mesh network. Int. J. Commun. Syst. 30(6), 1-15 (2017)

45. XD Song, X Wang, Extended AODV routing method based on distributed minimum transmission (DMT) for WSN. Int J Electron Commun 69(1), 371-381 (2015)

46. CP Zhao, A new medium access control protocol based on perceived data reliability and spatial correlation in wireless sensor network. Comput Electric Eng 38(3), 694-702 (2012)

47. JQ Chen, GQ Mao, Capacity of cooperative vehicular networks with infrastructure support: multi-user case. IEEE Trans. Veh. Technol. 67(2), 1546$1560(2018)$

\section{Submit your manuscript to a SpringerOpen ${ }^{\circ}$ journal and benefit from:}

- Convenient online submission

Rigorous peer review

- Open access: articles freely available online

- High visibility within the field

- Retaining the copyright to your article

Submit your next manuscript at $\gg$ springeropen.com 